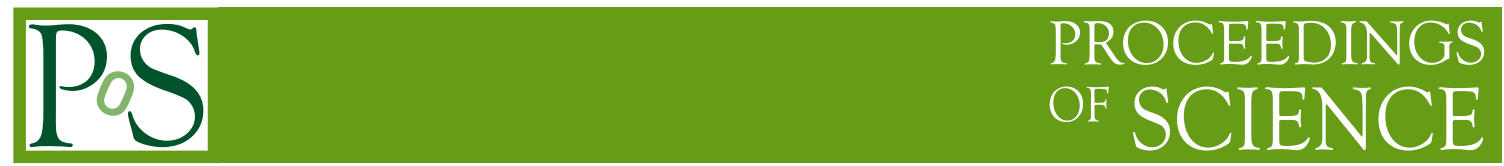

\title{
Hadron Spectroscopy Review
}

Christopher E. Thomas ${ }^{* \dagger}$

Trinity College Dublin

E-mail: thomascemaths.tcd.ie

I review recent lattice calculations of meson and baryon spectra. A selection of highlights are discussed with the focus mainly on studies of higher-lying/excited states and resonances or nearthreshold states. Some new calculations of lower-lying heavy-light and light isoscalar mesons are also mentioned.

31st International Symposium on Lattice Field Theory LATTICE 2013

July 29 - August 3, 2013

Mainz, Germany

\footnotetext{
* Speaker.

${ }^{\dagger}$ Current affiliation: DAMTP, University of Cambridge
} 


\section{Introduction}

Meson and baryon spectra provide probes of the non-perturbative regime of QCD. Historically, much of the pattern of mesons could be understood in terms of a quark-antiquark pair moving in a potential. However, more recently many states have been observed [1] that do not fit into this pattern, for example, the " $X, Y, Z$ 's" in charmonium, the charm-strange $D_{s 0}(2317)$ and the somewhat older puzzle of the light scalar mesons ${ }^{1}$. There have been many suggestions as to the nature of these states, for example, hybrid mesons where the gluonic field is excited or multiquark systems containing more quark-antiquark pairs (such as molecular states of mesons or tetraquarks containing two quarks and two antiquarks). States with exotic quantum numbers are particularly interesting, i.e. those which cannot arise from solely a quark-antiquark pair such as spin $(J)$, parity $(P)$, charge-conjugation $(C)$ combinations (e.g. $J^{P C}=0^{--}, 0^{+-}, 1^{-+}, 2^{+-}$) or exotic flavour states (e.g. charmonium-like states with non-zero charge). Experimentally, in the light meson sector there are suggestions of an exotic $1^{-+}$state [4] whereas in the charmonium and bottomonium systems there have been observations of charged structures but there are no hints of exotic $J^{P C}$. Many ongoing and planned experiments are investigating these topics including COMPASS, KLOE, BESIII, experiments at the LHC, GlueX and CLAS12 at Jefferson Lab, Belle II, and Panda at GSI/FAIR.

Moving to baryons there is the issue of 'missing' resonances: there are many more states expected than have been observed experimentally [1]. The question then arises as to whether these states exist and just have not yet been seen (perhaps because they are too wide or they couple weakly to the production mechanisms in current experiments) or whether they do not exist because there is a 'freezing' of degrees of freedom (for example, two quarks in the baryon form a diquark with internal degrees of freedom that, at least for the lighter states, are not excitable). Gluonic excitations can also be present with the resulting hybrid baryons leading to an even denser spectrum ${ }^{2}$. Then there is the puzzle of the first excitation in the $J^{P}=\frac{1}{2}^{+}$nucleon channel, the so-called Roper resonance, which appears at a lower mass than expected. There is much experimental interest in baryons with, as well as some of those listed above, current and upcoming experiments at JLab, GSI/FAIR and J-PARC, ELSA, MAMI and Spring-8.

Lattice QCD provides an opportunity to perform ab-initio computations of spectra and so to understand these puzzling observations and learn about the non-perturbative regime of QCD. There has recently been much progress in computing the properties of higher-lying and excited states, as well as in precision calculations of the properties of the ground states in each sector. In general, spectral information follows from considering a spectral decomposition of one or more two-point correlation functions, $C_{i j}(t)=\left\langle 0\left|\mathscr{O}_{i}(t) \mathscr{O}_{j}^{\dagger}(0)\right|\right\rangle$, featuring interpolating operators, $\mathscr{O}$, that efficiently create and annihilate the states of interest. For mesons the interpolating operators typically consist of fermion bilinears, $\sim \bar{\psi} \Gamma \psi$, containing a Dirac matrix $\Gamma$ (or a Pauli matrix in a non-relativistic formulation with two-component fermion fields) and for baryons they generally consist of three fermion fields $\sim \varepsilon^{a b c} \psi_{a} \psi_{b} \psi_{c}$ coupled antisymmetrically in colour $(a, b, c)$ and with various spin couplings possible. There may be displacements and/or derivatives acting on the fermion fields giving operators with different spatial structures. Typically the fields are also

\footnotetext{
${ }^{1}$ see e.g. Refs. [2, 3] for reviews

${ }^{2}$ though there are no smoking-gun exotic $J^{P C}$ quantum numbers because baryons are not eigenstates of chargeconjugation
} 
smeared to remove high-frequency modes and improve the overlap onto the states being considered. In addition there may be additional fermion/antifermion fields giving an operator that has a structure resembling a multiquark system or a product of two or more hadrons, something that will be discussed further in Section 2.2.

For the study of excited states, the variational method [5, 6, 7] (also known as the generalised eigenvalue problem) is in common usage though other approaches, such as Bayesian multiexponential fits, are also used. In brief, the variational method involves, for each quantum-number channel, considering a basis of $N$ operators and then constructing a matrix of of correlation functions, $C_{i j}(t)$, where $i, j=1,2, \ldots, N$. A generalised eigenvalue problem,

$$
C_{i j}(t) v_{j}^{(n)}=\lambda^{(n)}(t) C_{i j}\left(t_{0}\right) v_{j}^{(n)}
$$

is then solved for an appropriate reference time $t_{0}[8,7]$. The time-dependence of an eigenvalue $\lambda^{(n)}(t)$ is related to the energy of the $n^{\prime}$ th state and the eigenvector, $v_{i}^{(n)}$, is related to the operatorstate overlap, $Z_{i}^{(n)} \equiv\left\langle 0\left|\mathscr{O}_{i}\right| n\right\rangle$; these overlaps can be used to probe the structure of states. The eigenvectors also give the optimal (in a variational sense) linear combination of operators to create the $n$ 'th state. Although many studies follow the general approach outlined here, there are significant variations in the formulation of the lattice action and, importantly for the present discussion, the number and construction of interpolating operators.

In the remainder of this review I will highlight a selection of recent work, concentrating mainly on calculations of higher-lying and excited states in each sector. The scope will be limited to hadrons with baryon number of magnitude 0 and 1 (i.e. meson or baryon-like systems); multinucleon states and related topics are reviewed by A. Walker-Loud [9]. Many of the interesting structures have been observed at energies close to or above the threshold for strong decay into lighter hadrons and so I include some studies of resonances and near-threshold states; there will be further and complementary discussion of these areas in the review by M. Döring [10]. I discuss mesons in Section 2 followed by baryons in Section 3. Section 4 concludes with a brief summary and outlook.

\section{Mesons}

Before discussing studies of higher-lying states and resonances, I begin with some precision calculations of lower-lying stable mesons where relatively simple fermion-bilinear operators are used and all the systematics effects are under control. The HPQCD collaboration have recently updated their heavy-light meson calculations to include charm quarks in the sea [11]. Dynamical HISQ u,d,s and c quarks are used with a non-relativistic b quark (including $O\left(\alpha_{s}\right)$ corrections). Updated hyperfine splittings and a summary plot of the spectrum of 'gold-plated' mesons are given in Ref. [11] where the 'postdictions' are seen to be in good agreement with experimental results and several predictions are made. The inclusion of charm quarks in the sea has not produced any noticeable changes and the results are also in agreement with those from using HISQ b quarks. HPQCD have also investigated various precision tests of the $J / \psi$ : the mass, leptonic width and radiative decay rate to the $\eta_{c}[12]$.

Isoscalar (isospin=0) mesons have all flavour quantum numbers equal to zero and provide an interesting probe of the non-perturbative physics of QCD and annihilation dynamics. Lattice 
studies of this sector have historically been hampered by the difficulty of getting a good statistical signal for the disconnected contributions to the correlators. At this meeting the ETM collaboration presented new preliminary results on the $\eta$ and $\eta^{\prime}$ meson masses, decay constants and the $\eta-\eta^{\prime}$ mixing angle $[13,14]^{3}$ using dynamical twisted mass $\mathrm{u}, \mathrm{d}, \mathrm{s}$ and $\mathrm{c}$ quarks. They were able to improve the signals, particularly for the $\eta^{\prime}$, by assuming that the disconnected contributions are only large for the $\eta$ and $\eta^{\prime}$ and negligible for higher states, something that they test in explicit computations. The calculations were performed for a range of pion masses on three different lattice spacings and then extrapolated to the continuum and physical limit giving $M_{\eta}, M_{\eta^{\prime}}$ and a mixing angle all in agreement with experiment.

Figure 1 shows a compendium of results on the $\eta$ and $\eta^{\prime}$ masses from different groups. Some preliminary Hadron Spectrum Collaboration results are included ${ }^{4}$ which extend the results in [17] to additional pion masses and lattice volumes. 'Distillation' [18] was used to efficiently evaluate the disconnected contributions with high statistical precision and this enables the masses of many excited and high-spin states to be extracted, including those with exotic quantum numbers, and their hidden-flavour content to be determined.

\subsection{Excited 'single-meson' spectroscopy}

Moving to higher-lying mesons where in general not all systematic effects are under complete control, the Hadron Spectrum Collaboration has continued its programme of calculations on anisotropic lattices, which have a fine temporal lattice spacing, with dynamical Clover $\mathrm{u}, \mathrm{d}$ and s quarks. For each quantum number channel, a large basis of fermion-bilinear operators is constructed combining every possible Dirac gamma matrix with up to three gauge-covariant derivatives acting on the fermion field, resulting in various spin and spatial structures. 'Distillation' [18] is used to smear the fermion fields and enables the correlation functions featuring these many different operators to be computed efficiently. The variational method is then used to analyse the resulting matrices of correlation functions.

On a cubic lattice in a finite cubic volume, the three-dimensional rotational symmetry of the continuum is reduced to the cubic symmetry group [21] and so states are no longer labelled by spin, $J$, but by the appropriate irreducible representations of the cubic symmetry group. The Hadron Spectrum Collaboration has developed a method to identify the continuum spin of extracted states at a single lattice spacing. Operators are constructed which would overlap with only one $J$ in an infinite volume continuum; these are then subduced into the appropriate irreducible representations and operator-state overlaps are used to identify the continuum spin of each extracted state [22,23].

Following calculations of light meson spectra [22, 23, 17] and the spectrum of charmonium [24], the most recent results are extensive charm-light $(D)$ and charm-strange $\left(D_{S}\right)$ meson spectra [25] across all $J^{P}$ combinations with $J \leq 4$. Computations are performed on a couple of lattice volumes, at a single lattice spacing, with light quark masses corresponding to $M_{\pi} \approx 400 \mathrm{MeV}\left(N_{f}=2+1\right)$, and a relativistic Clover formulation is used for the charm quark. As explained in that reference, most of the states fit into the general pattern expected in quark models of a quark-antiquark pair moving in a potential where the quark and antiquark spins are coupled to total spin $S$, they have

\footnotetext{
${ }^{3}$ this work has subsequently appeared as [15]

${ }^{4}$ these have now appeared as [16]
} 


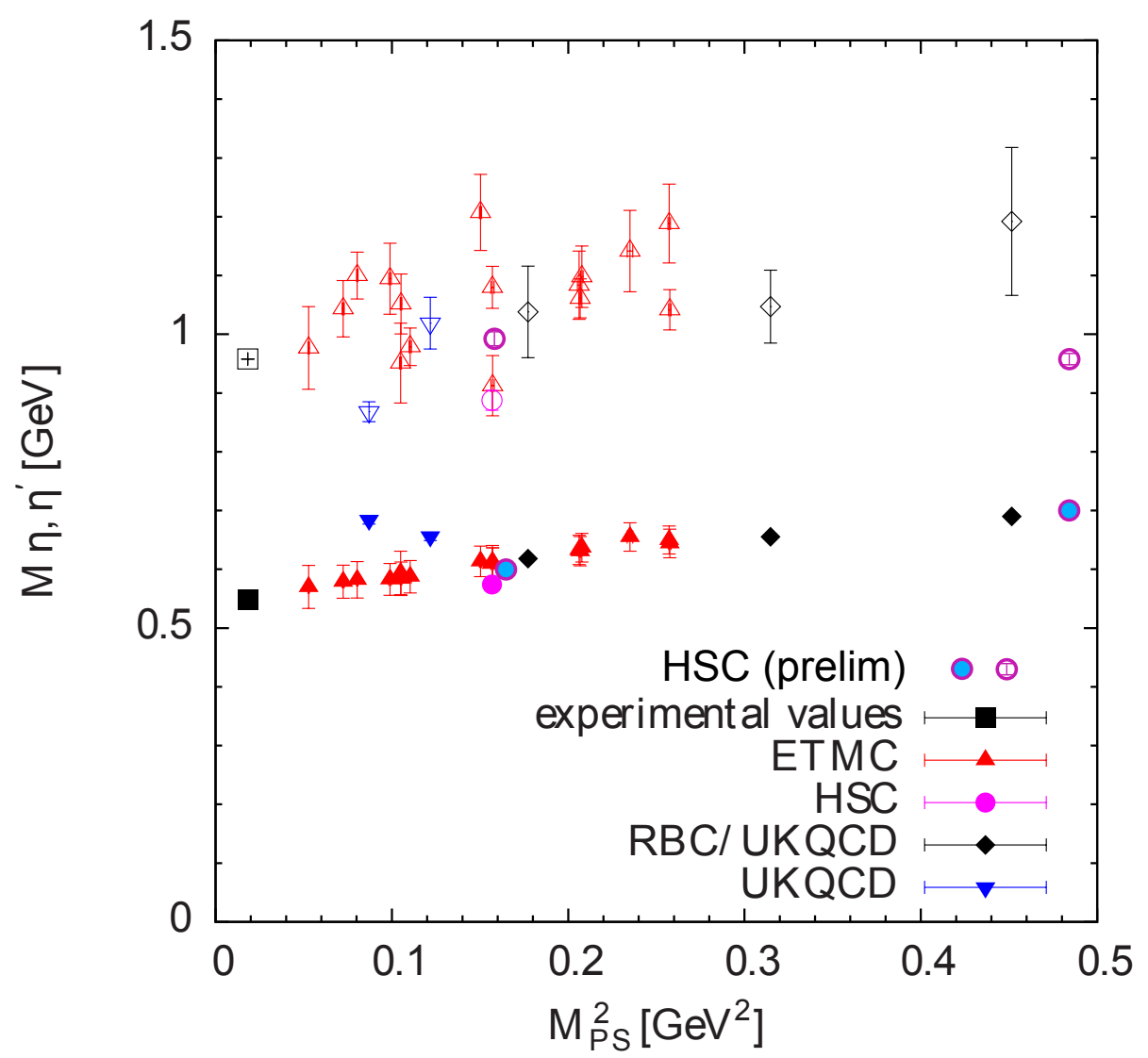

Figure 1: Slightly modified version of plot from C. Urbach [13]. Compendium of $\eta$ (filled symbols) and $\eta^{\prime}$ (open symbols) masses as a function of $M_{\pi}^{2}$ from ETMC [13, 14], HSC (Hadron Spectrum Collaboration) [17], RBC/UKQCD [19], UKQCD [20] and preliminary HSC results (which have now appeared in [16]). Also shown are the experimental masses.

relative orbital angular momentum $L$, and $L$ and $S$ are coupled to give total angular momentum $J$. However, there are a number of states which do not fit into this pattern and these are found to have a large overlap with operators featuring a commutator of two covariant derivatives, proportional to the field strength tensor. This suggests that the gluonic field plays an important role and these states are identified as hybrid mesons. The pattern of $J^{P}$ of the lightest hybrids ${ }^{5}\left(0^{-}, 1^{-}, 1^{-}, 2^{-}\right)$ is consistent with a colour-octet $1^{+-}$gluonic excitation coupled to a colour-octet quark-antiquark pair with $L=0$. These hybrids appear at an energy $\sim 1.2-1.3 \mathrm{GeV}$ above the lightest conventional state in the relevant flavour sector and further evidence that they all lie in the same lightest 'supermultiplet' of hybrid mesons comes from comparing operator-state overlaps. Interestingly, the same pattern and gluonic excitation energy scale were found in the light meson spectrum [26], in charmonium [24] and for baryons [27, 28], suggesting common physics. In charmonium a first excited supermultiplet of hybrid mesons was also identified and found to be consistent with the same gluonic $1^{+-}$but coupled to a quark-antiquark pair with $L=1$ - candidates for some of these states were found high up in the charm-light and charm-strange spectra but we do not robustly

\footnotetext{
${ }^{5}$ note that charm-light and charm-strange mesons are not eigenstates of charge conjugation and so there are no exotic $J^{P C}$ here in contrast to the light and charmonium sectors
} 
determine all the states in that energy region.

These computations also provide a probe of flavour symmetry breaking and mixing dynamics in QCD: the admixture of $S=0$ and $S=1$ states with the same $L$ and $J$ into the physical states - these would have opposite generalised $C$-parity, and so be forbidden to mix, if the quark and antiquark were degenerate. In [25] the mixing angles are found to lie between zero and the values expected in the heavy-quark limit, consistent with the charm quark having a mass at an intermediate scale.

Lattice calculations are performed in a finite box and hence the spectrum of multi-hadron states is discrete; those with the correct quantum numbers should appear in the spectral decomposition of the relevant correlators and so, in principle, should be present in the extracted spectra. However, when only fermion bilinear operators are used, we generally see no clear evidence for multi-hadron states [23]. Ref. [29] gives an explicit demonstration that, in order to reliably extract two-meson energy levels, operators with the correct structure, i.e. constructed from the product of two fermionbilinears, are required. The demonstration also justifies the statement that, when only fermion bilinear operators are used, the extracted mass of an unstable state will be within $\sim$ the hadronic width of the resonance mass. These considerations are relevant for the discussion in the following section: multi-hadron energy levels must be extracted reliably in order to study the properties of resonances on the lattice.

\subsection{Resonances and near-threshold states}

Most hadrons are unstable with respect to the strong interaction - they can decay strongly to two or more lighter hadrons. In particular, many of the unusual structures recently observed in the open and hidden charm and bottom sectors are close to or above the threshold for strong decay. Lattice computations of scattering observables and the properties of resonances are discussed in detail in the review by M. Döring [10] and here I will concentrate on a few examples of nearthreshold states and resonances that appear in elastic scattering channels.

Dynamical properties generally cannot be computed directly in the Euclidean formulation of lattice QCD. A relation between multi-hadron energies in a finite volume and the infinite volume scattering phase shift was derived by Lüscher [6,30] and has been extended by others (e.g. $[31,32,33,34])$. In brief, for elastic scattering and ignoring complications arising from the mixing of partial waves due the reduced symmetry of a finite box ${ }^{6}$, extracting an energy level with restframe energy $E_{\mathrm{cm}}$ gives the scattering phase shift $\delta$ at $E_{\mathrm{cm}}$. If many energy levels are extracted, the energy dependence of the phase shift can be mapped out and the scattering/resonance parameters determined from a fit to an appropriate function, for example an effective range expansion or a Breit-Wigner form. A point to note is that although the Lüscher method relies on computations being performed in a finite volume, corrections that are exponentially volume suppressed are neglected. It is therefore important not to use too small a volume and to check this, for example, by comparing results computed in different volumes.

Because the momenta in a finite volume are quantised, there are a limited number of energy levels that can be extracted in a given energy range even if many single and multi-hadron operators are used. Various strategies, all with advantages and disadvantages, have been employed to increase

\footnotetext{
${ }^{6}$ these are discussed in [10]
} 
the number of energies, and hence the number of phase shift points, extracted: considering the system at overall non-zero momentum (a 'moving frame'), performing computations in boxes of different sizes and shapes, and using twisted boundary conditions to gain access to different internal momenta. For a moving frame or a rectangular box the relevant symmetry is reduced further and so there is additional mixing between different partial waves. Performing calculations on many lattices of various sizes and/or shapes is generally computationally expensive. If twisted boundary conditions are only used in the valence sector, computations of some quantities may suffer from the formulation not being unitary; using twisted boundary conditions also in the sea increases the computational cost.

\subsubsection{The $\rho$ resonance}

One of the simplest resonances is the $\rho(770)$ that appears in $\pi \pi$ scattering in $L=1$ with $J^{P C}=1^{--}$and isospin $I=1$; empirically the $\rho$ decays almost entirely to $\pi \pi$ [1]. There have been number of dynamical $\left(N_{f}=2\right.$ or $\left.2+1\right)$ lattice studies with various pion masses that use different moving frames in order to extract a few phase shift points [35, 36, 37]. With a Breit-Wigner parameterization, reasonable values for the resonance mass and width can be extracted. However, because only a few phase shift points are extracted, it is not always obvious that a resonance, and only a single resonance, is present if this was not known in advance. In addition, the effect of mixing with higher partial waves has not been investigated. In [38] ( $\left.N_{f}=2, M_{\pi} \approx 300 \mathrm{MeV}\right)$, instead of using different moving frames, computations are performed in a cubic and two rectangular boxes in order to get points reasonably well distributed over the phase shift curve.

The Hadron Spectrum Collaboration has used many single-meson (fermion-bilinear) and $\pi \pi$ operators in various moving frames with a variational analysis in order to extract a large number of phase shift points [29]. Following the formalism developed in [39], the $\pi \pi$ operators are constructed as,

$$
\mathscr{O}_{\Lambda, \mu}^{\left[\left|\vec{p}_{1}\right|,\left|\vec{p}_{2}\right|\right]}(\vec{P})=\sum_{\vec{p}_{1}, \vec{p}_{2}} \mathscr{C}_{\Lambda, \mu}\left(\vec{P}, \vec{p}_{1}, \vec{p}_{2}\right) \mathscr{O}_{\pi}\left(\vec{p}_{1}\right) \mathscr{O}_{\pi}\left(\vec{p}_{2}\right)
$$

where $\mathscr{O}_{\pi}$ is a fermion-bilinear interpolating operator for a pion, $\vec{P}$ is the overall momentum, the sum over momenta $\vec{p}_{1}, \vec{p}_{2}$ related by an allowed lattice rotation is subject to the constraint $\vec{P}=\vec{p}_{1}+\vec{p}_{2}$, and $\mathscr{C}_{\Lambda}$ is a generalised Clebsch-Gordan coefficient that projects onto an appropriate irreducible representation, $\Lambda$, and row $\mu$, of the relevant symmetry group. To improve overlap onto $\pi \pi$, the $\mathscr{O}_{\pi}$ sub operators are the optimal linear combination of the fermion-bilinear operators discussed above that produce a ground state $\pi$ with the coefficients determined from a variational analysis. The distillation technique [18] allows the large number of correlation functions, including those featuring two-meson operators, to be computed efficiently, along with the required $t$-to- $t$ propagators and the various momentum projections at both the source and the sink. Many different irreducible representations were considered in order to constrain the effect of partial wave mixing and three volumes were used in order to obtain more points and to allow for a consistency check.

The results of this study are summarised in Figure 2 where it can be seen that the energy dependence of the phase shift has been mapped out in detail. The plot shows convincingly the rapid rise in the phase shift from $0^{\circ}$ through $90^{\circ}$ to $180^{\circ}$ that is expected in the presence of a single isolated resonance. The determined resonance mass is not too different from experiment but the width is significantly smaller - this is simply because the pion mass in this study is $\approx 400 \mathrm{MeV}$ and 


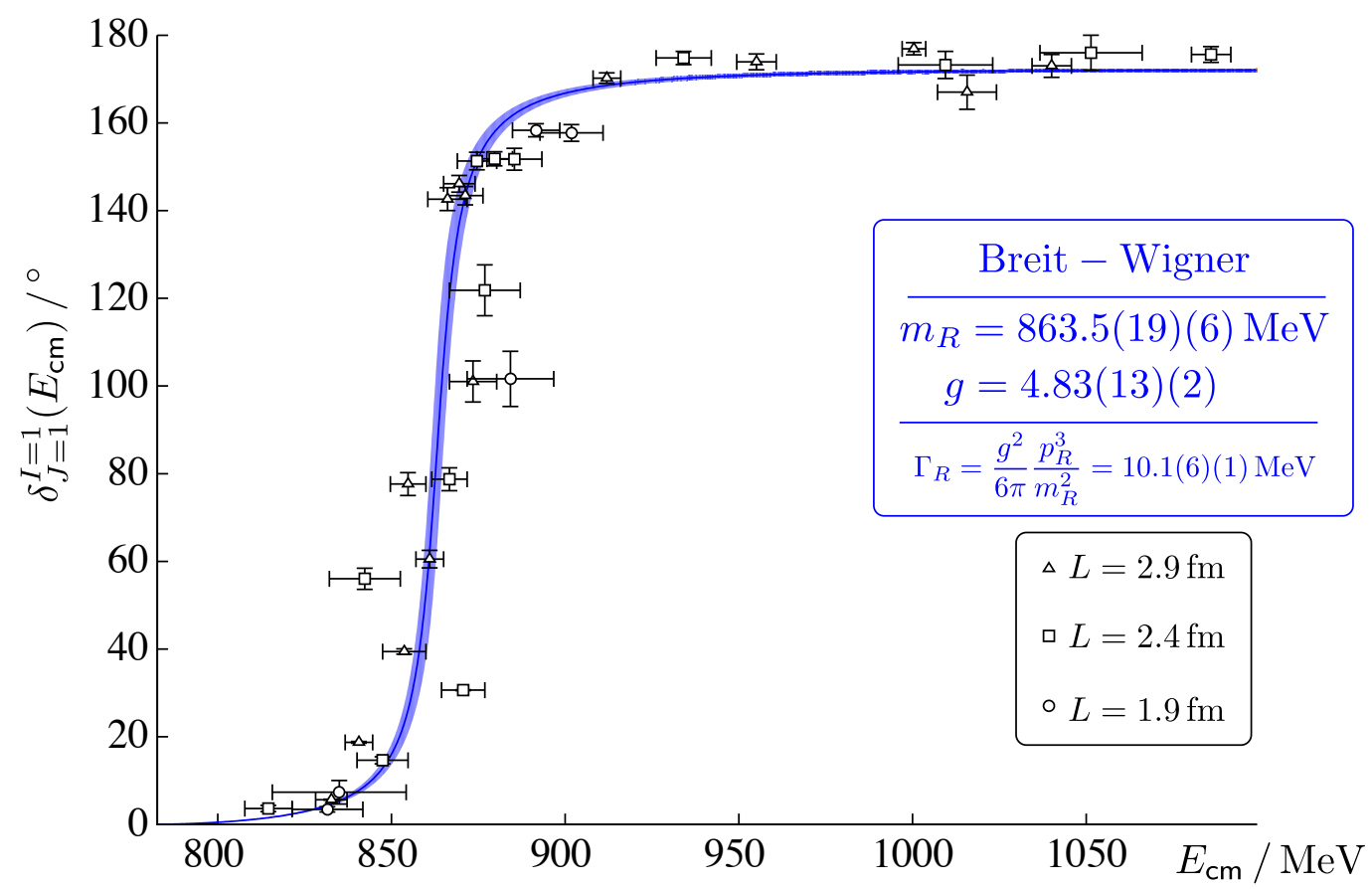

Figure 2: From [29]. The $I=1, L=1 \pi \pi$ elastic scattering phase shift plotted from $\pi \pi$ threshold to the inelastic $K \bar{K}$ threshold, from a dynamical calculation $\left(N_{f}=2+1\right)$ with $M_{\pi} \approx 400 \mathrm{MeV}$. Points are from an energy-level by energy-level analysis and the curve is from a global fit to a relativistic Breit Wigner parameterization as described in [29].

so the phase space for decay is much reduced; the coupling $g$ defined in the figure, where the phase space factor is removed, is more consistent with the experimental value. Perhaps such a detailed determination of the phase shift curve is not necessary for the relatively simple and well understood $\rho$ resonance. However, the ability to map out the energy dependence in detail will be vital in more complicated systems where the situation is phenomenologically less clear.

Although distillation provides an efficient way to compute many different correlation functions, the method suffers from a rapid increase in computational cost as the volume is enlarged and one possibly way to ameliorate this is to use a stochastic variation [40]. Some preliminary results from using stochastic distillation to compute correlation functions involving single-meson and many different types of two-meson operators in the $\rho$ and $K^{*}$ channels are presented in [41].

\subsubsection{Other channels}

There have been a number of first attempts at studying resonances and near-threshold states in other channels. Although these represent encouraging developments, they are not as mature as the $\rho$ computations and more work is necessary before detailed and robust conclusions can be drawn. Here I mention a few studies of charmonium and charmed meson channels. Other scattering channels in the light and strange meson sectors are discussed in [10]. 
In [42] $I=\frac{1}{2}$ resonances in $D \pi\left(0^{+}\right)$and $D^{*} \pi\left(1^{+}\right)$were investigated using $D$ (fermion bilinear) and $D^{(*)} \pi$ operators on a single ensemble $\left(N_{f}=2, M_{\pi}=266 \mathrm{MeV}\right)$ with a rather small volume. Distillation was used to compute the correlation functions and for each channel two or three points on the phase shift curve are extracted in the elastic region. Fits to a Breit Wigner yield the $0^{+}$and $1^{+}$resonance masses and widths, broadly consistent with experiment.

In [43] $]^{7}$ the charm-strange $D_{s 0}(2317)$ with $J^{P}=0^{+}$is studied using $D_{s}$ (fermion-bilinear) and $D K$ operators. Computations are performed on two different ensembles $\left(N_{f}=2, M_{\pi}=266 \mathrm{MeV}\right.$ and $N_{f}=2+1, M_{\pi}=156 \mathrm{MeV}$ ), both with a rather small volume, using distillation or stochastic distillation. Two energy levels are extracted in each case and the resulting phase shift points are found to correspond to a below-threshold state, in line with the experimentally observed $D_{s 0}(2317)$.

Another investigation [45] used a more indirect approach to study the $D_{s 0}(2317)$ and other charm-light and charm-strange mesons: different channels are related using the chiral unitary approach. Their results support the interpretation of the $D_{s 0}(2317)$ as a $D K$ molecule and they update a prediction for the isospin breaking decay width for $D_{s 0}(2317) \rightarrow D_{s} \pi$. At this meeting some preliminary Hadron Spectrum Collaboration results on $D K$ and $D \pi$ scattering were also presented [46].

Moving to the charmonium sector, a study of the $1^{++}$channel was presented at this meeting [47] where $c \bar{c}, D \bar{D}^{*}$ and $J / \psi \omega$ or $J / \psi \rho$ operators were used on one ensemble $\left(N_{f}=2\right.$, $M_{\pi}=266 \mathrm{MeV}$ ) with a small volume ${ }^{8}$. In the $I=0$ channel a couple of levels were extracted in the relevant energy region and found to correspond to a state just below threshold, perhaps a sign of the $X(3872)$. No evidence for a bound state or a resonance was found in the $I=1$ channel. Experimentally the $X(3872)$ lies very close to $D^{0} D^{* 0}$ threshold and slightly below $D^{+} D^{*-}$ threshold, and it shows significant isospin violation in its decays. This state will therefore be a challenge to understand quantitatively on the lattice requiring isospin breaking effects to be included and a delicate tuning of quark masses. A similar study, looking for the flavour-exotic $Z_{c}^{+}(3900)$ in $1^{+-}$ with $I=1$ using $D \bar{D}^{*}$ and $J / \psi \pi$ operators, found a weak interaction and no $Z_{c}^{+}(3900)$ candidate $^{9}$.

Another recent study in the charmonium sector used partially twisted boundary conditions to search for the $Y(4140)$ in $J / \psi \phi$ [50]. In computations on dynamical $\left(N_{f}=2+1\right)$ PACS-CS ensembles with $M_{\pi}=156 \mathrm{MeV}, S$ and $P$-wave scattering phase shifts were extracted and they found no sign of the $Y(4140)$.

\section{Baryons}

Moving to baryons, I begin with a recent study of the flavour structure of excited baryons before giving a compendium of results on excited nucleons and then mention some calculations of charmed baryon spectra. Baryon resonances are discussed in the review by M. Döring [10].

\subsection{Flavour structure of excited baryons}

The Hadron Spectrum Collaboration has recently computed very extensive excited spectra of light and strange baryons with all possible flavour combinations [28], extending earlier computations of non-strange $N$ and $\Delta$ baryon spectra [51, 27]. For each quantum number channel, the

\footnotetext{
${ }^{7}$ this has subsequently appeared as [44]

8 this has since appeared as [48]

9 this has now appeared as [49]
} 
distillation technique is used to compute two-point correlation functions involving a large number of three-quark operators with different spin and spatial structures (up to two gauge-covariant derivatives) giving access to $J \leq \frac{7}{2}$. These correlators are then analysed with the variational method and the continuum spin, $J$, of the extracted states is determined in a similar way as for mesons (see Section 2.1): operators are constructed to have a definite $J$ in the continuum and then subduced into irreducible representations of the relevant symmetry group; operator-state overlaps are used to identify the continuum spin.

Computations are performed on dynamical $\left(N_{f}=2+1\right)$ anisotropic lattices with a range of light quark masses corresponding to $M_{\pi} \approx 700 \mathrm{MeV}, 524 \mathrm{MeV}$ and $400 \mathrm{MeV}$, each with a single volume. The heaviest $\left(M_{\pi} \approx 700 \mathrm{MeV}\right)$ is the $\mathrm{SU}(3)$ flavour symmetric point where the up, down and strange quarks are degenerate; here the different flavour channels are labelled by their SU(3) symmetry as flavour singlet $\left(1_{F}\right)$, octet $\left(8_{F}\right)$ and decuplet $\left(10_{F}\right)$. When the light (up and down) quark masses are reduced, i.e. $\mathrm{SU}(3)$ flavour symmetry is broken, these classifications are no longer good and the physical baryon flavour multiplets are $\Lambda$ (mixture of $1_{F}$ and $\left.8_{F}\right), \Sigma\left(8_{F}\right.$ and $\left.10_{F}\right), \Xi\left(8_{F}\right.$ and $\left.10_{F}\right), \Omega\left(10_{F}\right)$ and the non-strange $N\left(8_{F}\right)$ and $\Delta\left(10_{F}\right)$. In each flavour channel, the spectra for $M_{\pi} \approx 524 \mathrm{MeV}$ and $400 \mathrm{MeV}$ are found to be basically superpositions of the relevant $\mathrm{SU}(3)$ multiplet spectra (for example, the $\Lambda$ spectrum is basically a superposition of the $1_{F}$ and $8_{F}$ spectra) with some mixing as determined by considering operator-state overlaps.

Throughout the extracted spectra, in each flavour sector the multiplicities of states in the lowest energy bands are as would be expected from a non-relativistic quark model, i.e. the number of states from $\mathrm{SU}(6) \times \mathrm{O}(3)$ (flavour $\times$ spin $\times$ space) symmetry considerations. In particular, there does not appear to be any freezing of degrees of freedom. There are additional states within the spectra that have a large overlap with operators featuring the field strength tensor and, as for mesons, these are identified as hybrids where the gluonic field is excited. The $J^{P C}$ pattern of the lightest hybrid baryons appears to be consistent with a colour-octet $1^{+-}$gluonic excitation coupled to colour octet $q q q$ and the scale of the excitation is $\sim 1.2-1.3 \mathrm{GeV}$; interestingly this is the same pattern and energy scale as found in the lattice calculations of the spectra of mesons with various flavours.

\subsection{Excited nucleons}

Now restricting the discussion to the $J=\frac{1}{2}$ nucleons $\left(I=\frac{1}{2}\right)$, many groups have studied the lower-lying states and compilations of recent results for positive and negative parity states are given in Figures 3 and 4 respectively; also shown are ' 3 '”' and ' $4 *$ ' states from the PDG review [1].

In the positive parity sector, Figure 3, it can be seen that the ground state nucleon mass tends towards the experimental value as the pion mass is reduced to the physical value. However, all the lattice calculations appear to miss the first excited state, the Roper, though some studies are at too high a pion mass or have too large an uncertainty to be able to draw a definite conclusion. There has been much discussion in the literature and at this meeting on this puzzle $[52,53,54,55$, $56]$ and it has been suggested that the P-wave $N \pi$, lying in this energy range, is relevant. Some calculations of excited nucleon wavefunctions relevant for this question are discussed in the review by S. Syritsyn [57]. The inclusion $N \pi$ operators along with three-quark operators in a variational analysis and computations in multiple volumes will be necessary to confirm the role of $N \pi$ and to determine the nature of the Roper. 


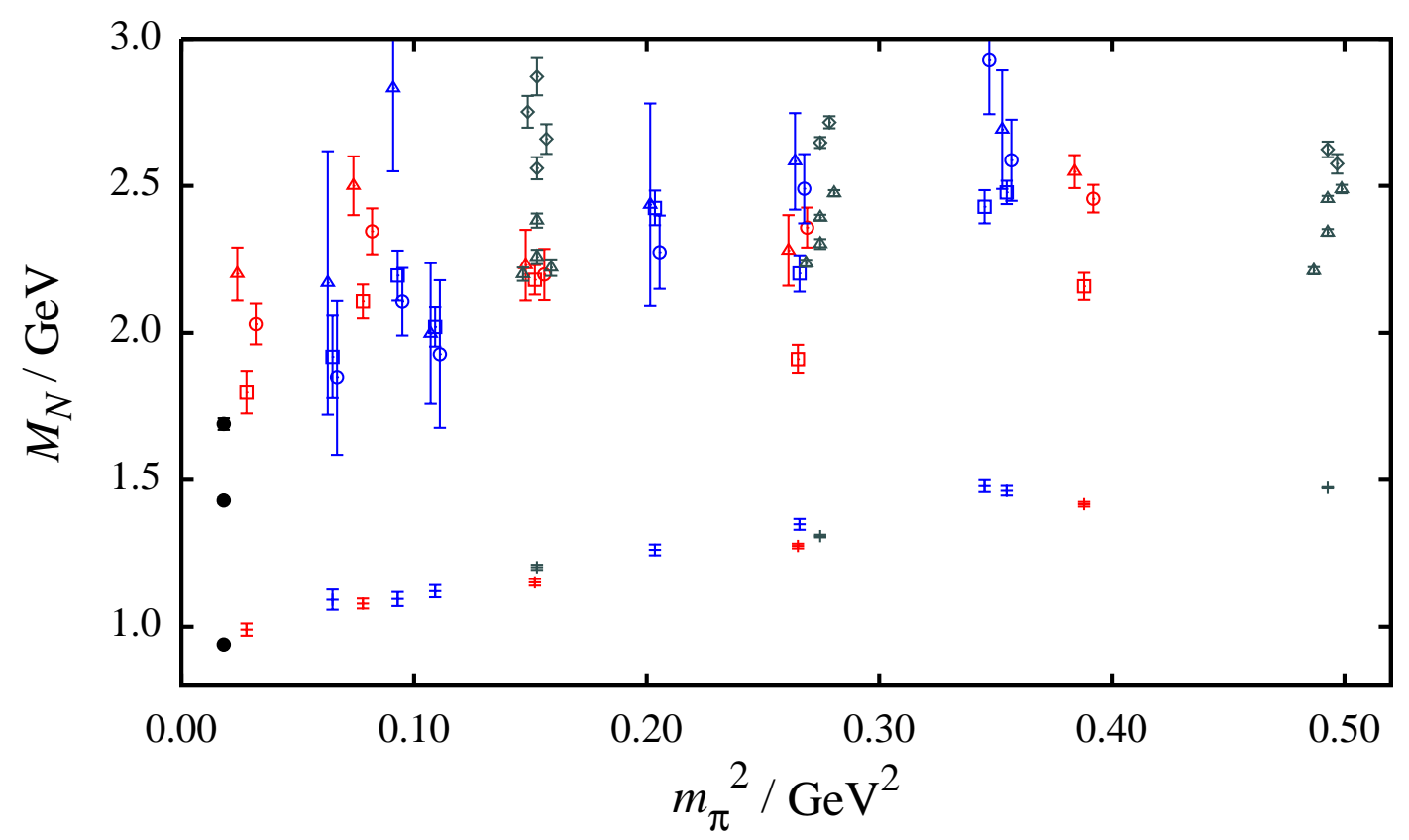

Figure 3: Compilation of some recent results on lower-lying $J^{P}=\frac{1}{2}^{+}$nucleons as a function of $M_{\pi}^{2}$ from BGR (open blue symbols) [52], CSSM (open red symbols) [53], Hadron Spectrum Collaboration (open grey symbols) [28], and experimental ' $3 *$ ' and ' $4 *$ ' states from the PDG review (solid black circles) [1]. Results at $M_{\pi}^{2} \sim 0.5 \mathrm{GeV}^{2}$ are at the $S U(3)$ flavour symmetric point. Note that some points have been displaced horizontally for clarity.

In the negative parity sector, Figure 4, the various computations show a lightest pair of states that are generally consistent with tending towards the two experimental masses shown. All the calculations appear to fail to find the S-wave $N \pi$ level, although BGR [52] interpret a significant change in the eigenvector structure at their lowest pion mass as the sign for an avoided level crossing. Again, the inclusion $N \pi$ operators along with three-quark operators in a variational analysis and computations in multiple volumes will be necessary to pin this down.

\subsection{Charm baryons}

Briefly moving to charmed baryons, the Hadron Spectrum Collaboration has, following the same lines as discussed in Section 3.1 above, recently computed an extensive spectrum of triplycharmed $\left(\Omega_{c c c}\right)$ baryons on dynamical $\left(N_{f}=2+1\right)$ ensembles with $M_{\pi} \approx 400 \mathrm{MeV}$. Again, the multiplicities in the lowest bands of states are consistent with what is expected in a non-relativistic quark model. Hybrid baryons are identified in the spectrum and the pattern again appears to be consistent with a colour-octet $1^{+-}$gluonic excitation coupled to colour octet $q q q$ and the scale of the excitation is $\sim 1.2-1.3 \mathrm{GeV}$. In addition, preliminary results on excited doubly-charmed baryons were presented at this meeting [58].

Recent studies of lower-lying charmed baryons presented at this meeting include Refs. [59, $60,61,62]$. 


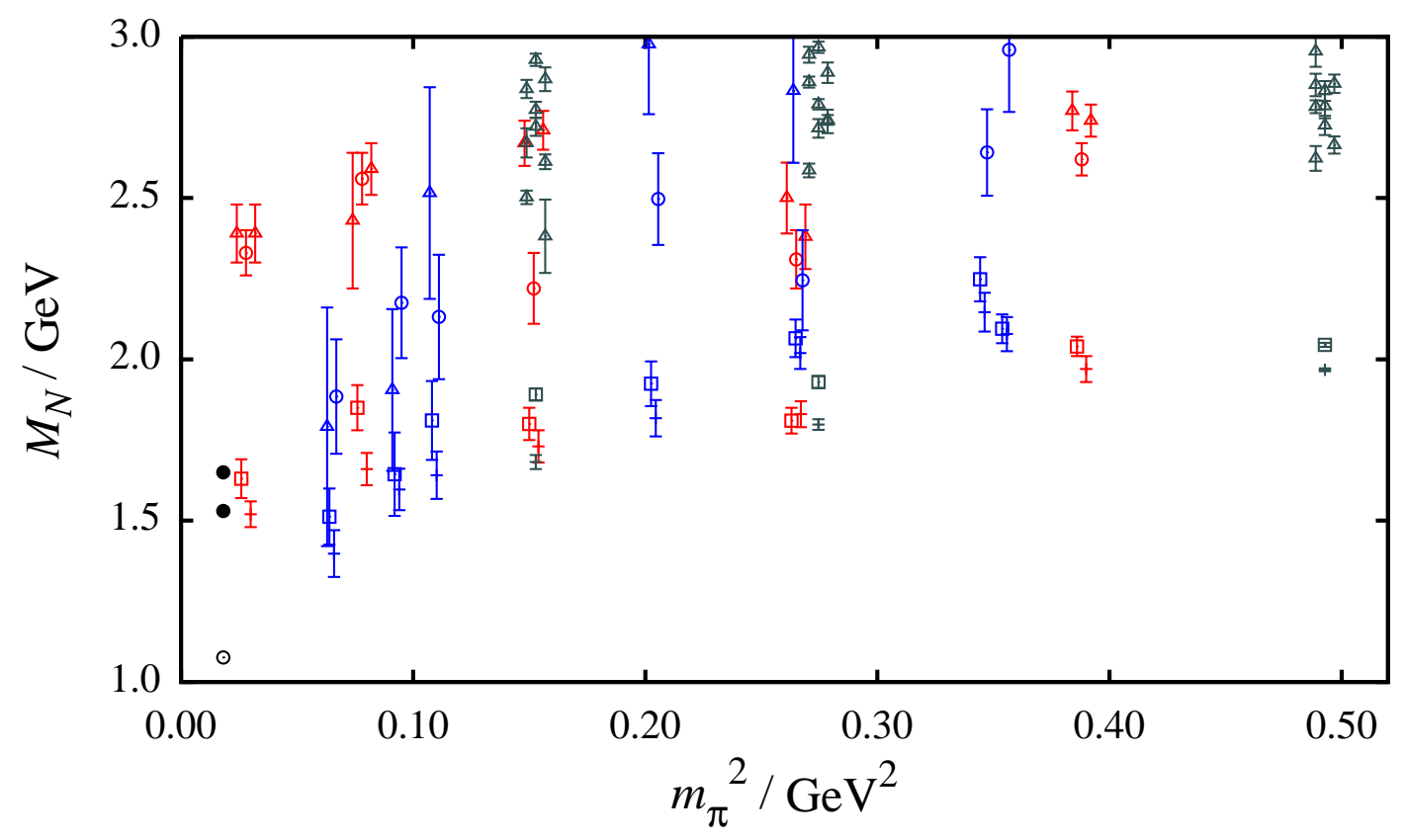

Figure 4: As Figure 3 but for $J^{P}=\frac{1}{2}^{-}$nucleons. The open black symbol at the physical $M_{\pi}^{2}$ is the noninteracting $N \pi$ threshold.

\section{Summary}

There has been significant progress in computing excited spectra on the lattice in recent years driven by the development of a number of techniques and computational advances (including the use of GPUs). Calculations have given insight into the non-perturbative regime of QCD: the flavour structure of hadrons, the relevant low energy degrees of freedom and a phenomenology of hybrid hadrons. These advances are complemented by the reduction of the various systematics leading to accurate and precise determinations of the lower-lying hadron masses in each sector.

However, there are many opportunities for further progress in lattice QCD calculations of hadron spectra. This is particularly true for investigations of resonances and near-threshold states which is an area that has recently been the focus of much work, something that looks set to continue. The relatively simple $\rho$ resonance has been studied in detail by many groups and, although first attempts have been made, there is still a lot of work to do for other channels. With further work it looks like lattice QCD can make progress in addressing some of the various puzzles in spectroscopy in the near future and we can learn more about the non-perturbative regime of QCD. Challenges in studying resonances and some possible strategies to overcome them are discussed in [10].

\section{Acknowledgments}

I thank the organisers of the meeting for the invitation to give this review. I would also like to thank everyone who sent me material, those who discussed their work with me and my colleagues in the Hadron Spectrum Collaboration, and to apologise to those whose work I don't cover. I ac- 
knowledge support from a Marie Curie International Incoming Fellowship, PIIF-GA-2010-273320, within the 7th European Community Framework Programme.

\section{References}

[1] J. Beringer et. al. (Particle Data Group), Review of Particle Physics (RPP), Phys. Rev. D86 (2012) 010001. and 2013 partial update for the 2014 edition.

[2] N. Brambilla, S. Eidelman, B. Heltsley, R. Vogt, G. Bodwin, et. al., Heavy quarkonium: progress, puzzles, and opportunities, Eur.Phys.J. C71 (2011) 1534, [arXiv:1010.5827].

[3] E. Klempt and A. Zaitsev, Glueballs, Hybrids, Multiquarks. Experimental facts versus QCD inspired concepts, Phys.Rept. 454 (2007) 1-202, [arXiv:0708.4016].

[4] C. Meyer and Y. Van Haarlem, The Status of Exotic-quantum-number Mesons, Phys.Rev. C82 (2010) 025208, [arXiv:1004.5516].

[5] C. Michael, Adjoint Sources in Lattice Gauge Theory, Nucl. Phys. B259 (1985) 58.

[6] M. Luscher and U. Wolff, How to calculate the elastic scattering matrix in two-dimensional quantum field theories by numerical simulation, Nucl. Phys. B339 (1990) 222-252.

[7] B. Blossier, M. Della Morte, G. von Hippel, T. Mendes, and R. Sommer, On the generalized eigenvalue method for energies and matrix elements in lattice field theory, JHEP 04 (2009) 094, [arXiv:0902.1265].

[8] J. J. Dudek, R. G. Edwards, N. Mathur, and D. G. Richards, Charmonium excited state spectrum in lattice QCD, Phys. Rev. D77 (2008) 034501, [arXiv: 0707 .4162].

[9] A. Walker-Loud, Nuclear physics review, PoS Lattice 2013 (2013) 013.

[10] M. Döring, Resonances and multi-particle states, PoS Lattice 2013 (2013) 006.

[11] R. Dowdall, C. Davies, T. Hammant, and R. Horgan, Precise heavy-light meson masses and hyperfine splittings from lattice QCD including charm quarks in the sea, Phys.Rev. D86 (2012) 094510, [arXiv:1207.5149].

[12] G. Donald, C. Davies, R. Dowdall, E. Follana, K. Hornbostel, et. al., Precision tests of the J/ $\psi$ from full lattice QCD: mass, leptonic width and radiative decay rate to $\eta_{c}$, Phys.Rev. D86 (2012) 094501, [arXiv:1208.2855].

[13] C. Urbach, $\eta$ and $\eta^{\prime}$ masses from lattice $Q C D$ with 2+1+1 quark flavours, PoS Lattice 2013 (2013) 252.

[14] K. Ottnad, Pseudoscalar flavor-singlet mixing angle and decay constants from $N_{f}=2+1+1$ WtmLQCD, PoS Lattice 2013 (2013) 253.

[15] C. Michael, K. Ottnad, and C. Urbach, $\eta$ and $\eta^{\prime}$ mixing from Lattice QCD, Phys.Rev.Lett. 111 (2013) 181602, [arXiv:1310.1207].

[16] J. J. Dudek, R. G. Edwards, P. Guo, and C. E. Thomas, Toward the excited isoscalar meson spectrum from lattice QCD, arXiv:1309.2608.

[17] J. J. Dudek et. al., Isoscalar meson spectroscopy from lattice QCD, Phys. Rev. D83 (2011) 111502, [arXiv:1102.4299].

[18] M. Peardon et. al. (Hadron Spectrum), A novel quark-field creation operator construction for hadronic physics in lattice QCD, Phys. Rev. D80 (2009) 054506, [arXiv: 0905.2160 ]. 
[19] N. Christ, C. Dawson, T. Izubuchi, C. Jung, Q. Liu, et. al., The $\eta$ and $\eta^{\prime}$ mesons from Lattice QCD, Phys.Rev.Lett. 105 (2010) 241601, [arXiv: 1002 .2999].

[20] E. B. Gregory, A. C. Irving, C. M. Richards, and C. McNeile (UKQCD Collaboration), A study of the eta and eta' mesons with improved staggered fermions, Phys.Rev. D86 (2012) 014504, [arXiv:1112.4384].

[21] R. C. Johnson, Angular momentum on a lattice, Phys. Lett. B114 (1982) 147.

[22] J. J. Dudek, R. G. Edwards, M. J. Peardon, D. G. Richards, and C. E. Thomas, Highly excited and exotic meson spectrum from dynamical lattice QCD, Phys. Rev. Lett. 103 (2009) 262001, [arXiv:0909.0200].

[23] J. J. Dudek, R. G. Edwards, M. J. Peardon, D. G. Richards, and C. E. Thomas, Toward the excited meson spectrum of dynamical QCD, Phys. Rev. D82 (2010) 034508, [arXiv: 1004.4930 ].

[24] L. Liu et. al. (for the Hadron Spectrum Collaboration), Excited and exotic charmonium spectroscopy from lattice QCD, JHEP 1207 (2012) 126, [arXiv: 1204.5425 ].

[25] G. Moir, M. Peardon, S. M. Ryan, C. E. Thomas, and L. Liu, Excited spectroscopy of charmed mesons from lattice QCD, JHEP 1305 (2013) 021, [arXiv:1301. 7670].

[26] J. J. Dudek, The lightest hybrid meson supermultiplet in QCD, Phys. Rev. D84 (2011) 074023, [arXiv:1106.5515].

[27] J. J. Dudek and R. G. Edwards, Hybrid Baryons in QCD, Phys.Rev. D85 (2012) 054016 , [arXiv:1201.2349].

[28] R. G. Edwards, N. Mathur, D. G. Richards, and S. J. Wallace, The Flavor Structure of the Excited Baryon Spectra from Lattice QCD, Phys.Rev. D87 (2013) 054506, [arXiv:1212.5236].

[29] J. J. Dudek, R. G. Edwards, and C. E. Thomas, Energy dependence of the $\rho$ resonance in $\pi \pi$ elastic scattering from lattice QCD, arXiv: 1212.0830.

[30] M. Luscher, Signatures of unstable particles in finite volume, Nucl. Phys. B364 (1991) 237-254.

[31] K. Rummukainen and S. A. Gottlieb, Resonance scattering phase shifts on a nonrest frame lattice, Nucl. Phys. B450 (1995) 397-436, [hep-lat/9503028].

[32] C. Kim, C. Sachrajda, and S. R. Sharpe, Finite-volume effects for two-hadron states in moving frames, Nucl.Phys. B727 (2005) 218-243, [hep-lat/ 0507006 ].

[33] N. H. Christ, C. Kim, and T. Yamazaki, Finite volume corrections to the two-particle decay of states with non-zero momentum, Phys.Rev. D72 (2005) 114506, [hep-lat/ 050700 9].

[34] L. Leskovec and S. Prelovsek, Scattering phase shifts for two particles of different mass and non-zero total momentum in lattice QCD, Phys.Rev. D85 (2012) 114507, [arXiv:1202.2145].

[35] X. Feng, K. Jansen, and D. B. Renner, Resonance Parameters of the rho-Meson from Lattice QCD, Phys. Rev. D83 (2011) 094505, [arXiv: 1011 .5288].

[36] C. Lang, D. Mohler, S. Prelovsek, and M. Vidmar, Coupled channel analysis of the rho meson decay in lattice QCD, Phys.Rev. D84 (2011) 054503, [arXiv:1105.5636].

[37] S. Aoki et. al. (CS Collaboration), $\rho$ Meson Decay in 2+1 Flavor Lattice QCD, Phys.Rev. D84 (2011) 094505, [arXiv:1106.5365].

[38] C. Pelissier and A. Alexandru, Resonance parameters of the rho-meson from asymmetrical lattices, Phys.Rev. D87 (2013) 014503, [arXiv: 1211 . 0092]. 
[39] J. J. Dudek, R. G. Edwards, and C. E. Thomas, S and D-wave phase shifts in isospin-2 pi pi scattering from lattice QCD, Phys. Rev. D86 (2012) 034031, [arXiv:1203.6041].

[40] C. Morningstar et. al., Improved stochastic estimation of quark propagation with Laplacian Heaviside smearing in lattice QCD, Phys. Rev. D83 (2011) 114505, [arXiv: 1104.3870 ].

[41] J. Bulava, B. Fahy, J. Foley, Y.-C. Jhang, K. J. Juge, et. al., Spectrum of excited states using the stochastic LapH method, PoS Lattice 2013 (2013) 266, [arXiv: 1310 . 7887].

[42] D. Mohler, S. Prelovsek, and R. Woloshyn, D $\pi$ scattering and $D$ meson resonances from lattice QCD, Phys.Rev. D87 (2013), no. 3 034501, [arXiv:1208.4059].

[43] D. Mohler, $d k$ scattering and the $d_{s}$ spectrum, PoS Lattice 2013 (2013) 240.

[44] D. Mohler, C. Lang, L. Leskovec, S. Prelovsek, and R. Woloshyn, The $D_{s 0}^{*}(2317)$ and DK scattering from lattice QCD, arXiv:1308.3175.

[45] L. Liu, K. Orginos, F.-K. Guo, C. Hanhart, and U.-G. Meissner, Interactions of Charmed Mesons with Light Pseudoscalar Mesons from Lattice QCD and Implications on the Nature of the $D_{s 0}^{*}(2317)$, Phys.Rev. D87 (2013) 014508, [arXiv:1208.4535].

[46] G. Moir, Excited spectroscopy of mesons containing charm quarks from lattice qcd, PoS Lattice 2013 (2013) 242.

[47] S. Prelovsek, L. Leskovec, and D. Mohler, Charmonium-like states from scattering on the lattice, PoS Lattice 2013 (2013) 254, [arXiv: 1310 . 8127].

[48] S. Prelovsek and L. Leskovec, Evidence for X(3872) from DD* scattering on the lattice, Phys.Rev.Lett. 111 (2013) 192001, [arXiv:1307.5172].

[49] S. Prelovsek and L. Leskovec, Search for $Z_{c}^{+}(3900)$ in the $1^{+}-$channel on the lattice, arXiv:1308.2097.

[50] S. Ozaki and S. Sasaki, Lúscher's finite size method with twisted boundary conditions: an application to J/ $\psi$ - $\phi$ system to search for narrow resonance, Phys.Rev. D87 (2013) 014506, [arXiv:1211.5512].

[51] R. G. Edwards, J. J. Dudek, D. G. Richards, and S. J. Wallace, Excited state baryon spectroscopy from lattice QCD, Phys. Rev. D84 (2011) 074508, [arXiv:1104.5152].

[52] G. P. Engel, C. Lang, D. Mohler, and A. Schafer, QCD with Two Light Dynamical Chirally Improved Quarks: Baryons, Phys.Rev. D87 (2013) 074504, [arXiv:1301.4318].

[53] M. S. Mahbub, W. Kamleh, D. B. Leinweber, P. J. Moran, and A. G. Williams, Structure and Flow of the Nucleon Eigenstates in Lattice QCD, Phys.Rev. D87 (2013) 094506, [arXiv: 1302 . 2987].

[54] D. S. Roberts, W. Kamleh, and D. B. Leinweber, Wave Function of the Roper from Lattice QCD, arXiv:1304.0325.

[55] W. Kamleh, Exploring the roper resonance in lattice qcd, PoS Lattice 2013 (2013) 245.

[56] K. Liu, The roper puzzle, PoS Lattice 2013 (2013) 246.

[57] S. Syritsyn, Hadron structure review, PoS Lattice 2013 (2013) 009.

[58] P. Madanagopalan, R. Edwards, N. Mathur, and M. Peardon, Spectroscopy of doubly and triply-charmed baryons from lattice qcd, PoS Lattice 2013 (2013) 247.

[59] R. A. Briceno, H.-W. Lin, and D. R. Bolton, Charmed-Baryon Spectroscopy from Lattice QCD with $N_{f}=2+1+1$ Flavors, Phys.Rev. D86 (2012) 094504, [arXiv: 1207.3536$]$. 
[60] Y. Namekawa et. al. (PACS-CS Collaboration), Charmed baryons at the physical point in $2+1$ flavor lattice QCD, Phys.Rev. D87 (2013) 094512, [arXiv:1301.4743].

[61] Z. Brown, W. Detmold, S. Meinel, and K. Orginos, Charmed bottom baryon spectroscopy, PoS Lattice 2013 (2013) 248.

[62] R. Horsley, J. Najjar, Y. Nakamura, H. Perlt, D. Pleiter, P. Rakow, G. Schierholz, A. Schiller, H. Stuben, and J. Zanott, Su(3) flavour symmetry breaking and charmed states, PoS Lattice 2013 (2013) 249. 